\title{
Reconciliation of Two Cognitive Models in Obsessive-Compulsive Disorder: An fMRI Study
}

\author{
Seungho Kim ${ }^{1 *}$, Sang Won Lee ${ }^{2,3 *}$, Hyunsil Cha1 ${ }^{1}$ Eunji Kim¹, Yongmin Chang ${ }^{1,4,5}$, and Seung Jae Lee ${ }^{3,6} \bowtie$ \\ ${ }^{1}$ Department of Medical \& Biological Engineering, Kyungpook National University, Daegu, Republic of Korea \\ ${ }^{2}$ Department of Psychiatry, Kyungpook National University Chilgok Hospital, Daegu, Republic of Korea \\ ${ }^{3}$ Department of Psychiatry, School of Medicine, Kyungpook National University, Daegu, Republic of Korea \\ ${ }^{4}$ Department of Molecular Medicine, School of Medicine, Kyungpook National University, Daegu, Republic of Korea \\ ${ }^{5}$ Department of Radiology, Kyungpook National University Hospital, Daegu, Republic of Korea \\ ${ }^{6}$ Department of Psychiatry, Kyungpook National University Hospital, Daegu, Republic of Korea
}

Objective Although cognitive models of obsessive-compulsive disorder (OCD) fall into two categories: cognitive deficit models and dysfunctional belief models, these approaches have their own ways and have hardly been reconciled. Therefore, this study aimed to investigate the potential relationships between cognitive deficit (using the Wisconsin Card Sorting Task, WCST) and dysfunctional belief (measured by scales of dysfunctional beliefs) mediated by neural activity in OCD patients.

Methods Thirty OCD patients and 30 healthy participants performed the WCST condition and a baseline MATCH condition during the 3T-functional magnetic resonance imaging (fMRI) acquisition.

Results Engagement of additional frontoparietal networks with poorer performance of WCST was found during the fMRI scan in OCD patients. Selected regions of interest from activated regions have positive relationships with dysfunctional beliefs and with the unacceptable thoughts symptom dimension in the OCD group.

Conclusion Findings suggest that alteration in frontoparietal networks related to cognitive deficits can be associated with dysfunctional beliefs while performing conventional neurocognitive tasks and this association with dysfunctional beliefs may be pronounced in the unacceptable thoughts domain-dominant OCD patients.

Psychiatry Investig 2021;18(6):545-552

Key Words Cognitive deficit, Executive function, Dysfunctional beliefs, Frontoparietal network, Obsessive-compulsive symptom domain.

\section{INTRODUCTION}

Since the 1980s, several cognitive models have been developed as general models of obsessive-compulsive disorder (OCD). ${ }^{1}$ Many researchers have conducted studies for elucidating cognitive abnormalities concerning the etiology and maintenance of OCD. ${ }^{2}$ Broadly, cognitive models fall into two

Received: September 13, 2020 Revised: February 10, 2021

Accepted: April 1, 2021

$\triangle$ Correspondence: Yongmin Chang, PhD

Department of Molecular Medicine, School of Medicine, Kyungpook National University, 680 Gukchaebosang-ro, Jung-gu, Daegu 41944, Republic of Korea Tel: +82-53-420-5471, Fax: +82-53-422-2677, E-mail: ychang@knu.ac.kr

$\triangle$ Correspondence: Seung Jae Lee, MD, PhD

Department of Psychiatry, School of Medicine, Kyungpook National University, 680 Gukchaebosang-ro, Jung-gu, Daegu 41944, Republic of Korea Tel: +82-53-200-5752, Fax: +82-53-426-5361, E-mail: jayleemd@knu.ac.kr

*These authors contributed equally to this work.

(a) This is an Open Access article distributed under the terms of the Creative Commons Attribution Non-Commercial License (https://creativecommons.org/licenses/bync/4.0) which permits unrestricted non-commercial use, distribution, and reproduction in any medium, provided the original work is properly cited. categories: cognitive deficit models, which propose that $\mathrm{OCD}$ is characterized by deficits in neuropsychological and information-processing functioning; and dysfunctional belief models, which postulate that OC symptoms arise from particular types of dysfunctional beliefs or appraisals. ${ }^{3}$ However, these two approaches have their own way and have hardly been reconciled. ${ }^{2}$

Previous studies on cognitive deficits have demonstrated that individuals with OCD show reduced performance on cognitive tasks such as executive functioning, cognitive inhibition, attention, and memory., ${ }^{4,5}$ This line of research relies on behavioral measures such as reaction time and neuropsychological assessment. ${ }^{6}$ In addition, these cognitive tasks have easily been used as cognitive activation paradigms in neuroimaging studies, and consequently, have greatly contributed to the development of the current neurobiological model of OCD. ${ }^{7}$ However, cognitive deficit models have their own limitations, wherein such impairments in these cognitive func- 
tions do not fully explain clinical symptoms, ${ }^{8}$ and could be caused by the effects of anxiety or fear which characterize OCD. They also do not account for the heterogeneity of OCD symptoms or their specificity, which differs from many psychiatric disorders that present mild cognitive deficits. ${ }^{2}$

By contrast, cognitive appraisal models propose that obsession and compulsions arise from specific types of dysfunctional beliefs and accordingly, the content of dysfunctional beliefs influences the types of OC symptoms. ${ }^{9,10}$ The Obsessive-Compulsive Cognitions Working Group has outlined three domains of dysfunctional beliefs including 1) over-estimation of threat and inflated responsibility, 2) importance of and need to control thoughts, and 3) perfectionism and intolerance of uncertainty. ${ }^{11,12}$ Unlike cognitive deficits, research on dysfunctional beliefs relies on self-reporting. ${ }^{2}$ As a result, the scientific adequacy of the appraisal models has been criticized. ${ }^{6}$ In fact, cognitive appraisal models have been developed in a way that has largely ignored the mounting body of research concerning the importance and role of neurobiological factors in OCD. ${ }^{1}$

However, some researchers have argued that cognitive deficits, dysfunctional beliefs, and metacognitive beliefs are likely interconnected. For instance, certain beliefs, such as thoughtaction fusion (TAF) cause people to dwell on potential threats, which in turn, could foster attentional biases or memory biases to address threats. ${ }^{2,13}$ Cognitive appraisals such as self-conscious beliefs could interfere with performance on neurocognitive tasks measuring cognitive deficits, thus perhaps leading patients with OCD to respond compulsively to achieve certainty. ${ }^{2,6}$ However, to our knowledge, no studies have specifically elucidated that different cognitive biases could affect or interact with one another. ${ }^{2}$ Getting a better understanding of the relationship between these two cognitive areas may help us understand how they might complement each other and offer a more comprehensive cognitive model of OCD.

The current study aimed to investigate the potential relationship between cognitive deficits, which were assessed by a computerized Wisconsin Card Sorting Test (WCST), and dysfunctional beliefs, which were measured by questionnaires. We chose the WCST as a measure of cognitive deficit because neural correlates of cognitive inflexibility or executive dysfunction have been well studied and closely relate to the current neurobiological model of OCD. ${ }^{7}$ We hypothesized that patients with OCD, relative to healthy controls (HC), would show poorer performance on the $\mathrm{WCST}^{5}$ and experience activation in additional areas of the brain., ${ }^{414}$ Then, we tested possible correlations between brain activity in differentially activated regions and dysfunctional beliefs in patients with OCD.

\section{METHODS}

\section{Participants}

Thirty patients with OCD (27 male and 3 female) and 30 healthy volunteers ( 29 male and 1 female) participated in this study. All participants were right-handed and between the ages of 18 and 35 years. For determining the presence of $\mathrm{OCD}$ and other comorbidities, a Structured Clinical Interview for DSM-5 Disorders, Clinical Version (SCID-5-CV) was carried out. Patients were excluded if they had a current comorbid Axis I diagnosis, psychotic symptoms, mental retardation, neurological disease, or a history of head injury or medical illness with documented cognitive sequelae. For healthy participants, psychiatric interviews were conducted for the exclusion of existing psychiatric pathology, psychotic symptoms, mental retardation, neurological disease, and history of head injury or medical illness. All interviews were completed by two experienced psychiatrists (S.W.L. and S.J.L.). All participants provided written informed consent according to the procedures approved by the Institutional Review Board of Kyungpook National University Hospital (2018-04-029).

\section{Psychological measures}

Clinical symptoms were assessed using the Obsessive-Compulsive Inventory-Revised (OCI-R) ${ }_{1}^{15}$ Dimensional ObsessiveCompulsive Scale (DOCS), ${ }^{16}$ and Beck Depression Inventory (BDI). ${ }^{17}$ Dysfunctional beliefs including guilt and importance of thoughts and responsibility were measured using the Guilt Inventory and Obsessive Beliefs Questionnaire-44 (OBQ-44), respectively. ${ }^{18}$

\section{Computerized Wisconsin Card Sorting Test}

All participants performed a modified version of the WCST during fMRI scanning. This paradigm was adapted from previous reports (Supplementary Figure 1 in the online-only Data Supplement)..$^{19}$ The task paradigm was a randomized block design, composed of the original WCST condition and a baseline MATCH condition. Instruction words were given for each condition: 'Card' for the WCST and 'Identical card' for MATCH condition. After the instruction word, participant performed the trial task following the rules of each condition and a feedback of response was shown as 'O' for a correct response or ' $\mathrm{X}$ ' for an incorrect response on the screen. Four reference cards and one target card were presented on the screen during the trial task. The target card was characterized from the four reference cards by three categories based on color, shape, and number. The participant was asked to find the correct category by selecting one of four reference cards according to characteristics of a target card in the WCST condition, and was asked to select a card that matches the target card within the 
four reference cards in the MATCH condition. If the participant correctly sorts the cards four times in a row in the WCST condition, a new category is created without instructions (set shifting). In addition, participants were measured based on their perseverative error score, which is the number of errors that the participant has chosen in the same category as the previous choice.

\section{MRI data acquisition and analyses}

All structural and functional imaging data was obtained via a 3.0 Tesla 750W MRI scanner (GE Healthcare, Milwaukee, WI, USA) with a 24 channel head and neck coil. A 3D brain volume imaging sequence was used to acquire structural brain image data [repetition time $(\mathrm{TR})=8.5 \mathrm{~ms}$, echo time $(\mathrm{TE})=3.2$ ms, flip angle $(F A)=12$-degree, matrix size $=256 \times 256$, and field of view $(\mathrm{FOV})=25.6 \mathrm{~cm}^{2}$ ]. The gradient echo planer $\mathrm{T} 2 *$ weighted imaging was used for acquiring functional image data $(\mathrm{TR}=$ $3,000 \mathrm{~ms}, \mathrm{TE}=30 \mathrm{~ms}, \mathrm{FA}=90$-degree, matrix size $=64 \times 64$, and $\mathrm{FOV}=23.0 \mathrm{~cm}^{2}$ ). All functional image data were analyzed using a statistical parametric mapping toolbox (SPM12; http:// www.fil.ion.ucl.ac.uk/spm), and statistical package for social sciences (SPSS25; http://www.ibm.com/analytics/spss-statistics-software). Preprocessing steps included realignment, slice timing, co-registration, normalization to the Montreal Neurological Institute (MNI) space, and smoothing (FWHM=8 mm). The motion parameter and outlier time series were detected using the artifact detection tools. The global signal change of 5 sigma and motion of $9 \mathrm{~mm}$ were thresholded for removing significant motion effects. The motion parameter and outlier effect were included as covariate factor in the first-level analysis. After first-level analysis was performed on individual images based on general linear model, full factorial ANOVA analysis was performed on each condition and group. The main effect of conditions was thresholded at $\mathrm{p}<0.05$, false discovery rate (FDR) corrected level across whole brain (Bonferroni correction was performed for main effects including WCSTMATCH or MATCH-WCST). Additional group comparison analysis in WCST condition was conducted with thresholded at $\mathrm{p}<0.05$, FDR corrected level for multiple comparisons. Regions of interest (ROIs) were created at locations of gray matter having maximum T-values on the conjunction map between main effect of condition and group comparison. After spheres $(7 \mathrm{~mm})$ were created at the location, the final ROIs were created by multiplying between spheres and activated regions on the conjunction map. The beta values were extracted using the REX toolbox (https://www.nitrc.org/projects/rex) on the conjunction map. Partial correlation analysis was applied between beta values and psychological measures after controlling for the effects of depression.

\section{RESULTS}

\section{Demographic and psychological information}

The demographic variables for each group are presented in Table 1. There were no significant differences between the groups

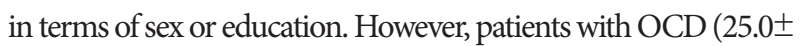
$5.2)$ were older than the healthy controls $(22.8 \pm 2.1)$. The OCD group demonstrated significantly greater scores for symptoms of OC, depression, and dysfunctional beliefs such as responsibility, importance of thoughts, feelings of guilt (all $\mathrm{p}<0.001$ ). The OCI revealed mild to moderate levels of severity in the OC group. Eleven (37\%) patients were drug naïve or had been drug-free for three months while 19 (63\%) patients were taking Selective Serotonin Reuptake Inhibitors (SSRIs), mostly escitalopram (Supplementary Table 1 in the online-only Data Supplement).

\section{Behavioral data}

Patients with OCD exhibited poorer performances than healthy individuals in the WCST condition; they had lower mean scores in numbers of response accuracy and set shifting yet higher perseverative errors in the WCST condition. However, there were no differences in accuracy in the MATCH condition (Figure 1).

\section{Effects of group and WCST condition in ANOVA analyses}

Full factorial ANOVA analysis showed there was a significant main effect of condition and group but no interaction effect. Post-hoc analysis revealed that the main effect of condition was exclusively explained by the contrast of WCST> MATCH, in which frontoparietal network, salience network (anterior insula, caudate, putamen, and thalamus), occipital gyri, lingual gyri, brain stems, and cerebellum (FDR corrected $\mathrm{p}<0.05$ ) (Figure 2, Supplementary Table 2 in the online-only Data Supplement) were activated. However, there were no significant brain regions in contrast of WCST $<\mathrm{MATCH}$.

\section{Group comparison analyses in WCST condition}

Within the WCST condition, group comparison analysis revealed that patients with OCD showed higher activations mostly in frontal and parietal regions, especially a right-side dominant pattern compared to the HC group (Figure 3 and Supplementary Table 3 in the online-only Data Supplement). However, the HC group showed no greater brain activations than the OCD group. Although statistical power of group differences was decreased after controlling for the effect of depression in a group comparison analysis (uncorrected $\mathrm{p}<0.001$ ), the initial pattern of group differences were still maintained (Supplementary Figure 2 in the online-only Data Supplement). 


\section{Brain activity vs. WCST performance, dysfunctional beliefs, and OC symptom dimensions}

Conjunction map was used to select WCST related brain ROIs in OCD patients. Conjunction map between WCST>
MATCH map in ANOVA analyses and OCD $>$ HC map of WCST condition in group comparison analyses revealed activation in right medial frontal gyrus, bilateral dorsolateral prefrontal cortices, right inferior orbito-frontal gyrus, right

Table 1. Demographic and clinical characteristics of patients with obsessive-compulsive disorder and healthy comparison subjects (mean \pm SD)

\begin{tabular}{|c|c|c|c|c|}
\hline \multirow{2}{*}{ Characteristics } & \multirow{2}{*}{$\begin{array}{l}\text { OCD } \\
\mathrm{N}=30\end{array}$} & \multirow{2}{*}{$\begin{array}{c}\mathrm{HC} \\
\mathrm{N}=30\end{array}$} & \multicolumn{2}{|c|}{ Statistics } \\
\hline & & & $\mathrm{t}$ & $\mathrm{p}$ \\
\hline Age, years & $25.0 \pm 5.2$ & $22.8 \pm 2.1$ & 2.2 & 0.037 \\
\hline Male/female & $27 / 3$ & $29 / 1$ & & $0.612^{*}$ \\
\hline Level of education, years & $14.5 \pm 1.7$ & $14.6 \pm 1.2$ & -0.3 & 0.792 \\
\hline Age at onset of OCD, years & $18.9 \pm 3.4$ & - & - & - \\
\hline Duration of illness, years & $5.8 \pm 4.2$ & - & - & - \\
\hline \multicolumn{5}{|l|}{ Symptom measures } \\
\hline OCI, total & $35.1 \pm 14.1$ & $11.5 \pm 5.5$ & 8.5 & $<0.001$ \\
\hline \multicolumn{5}{|l|}{ DOCS } \\
\hline Germs and contamination & $6.5 \pm 4.9$ & $2.9 \pm 1.7$ & 3.7 & $<0.001$ \\
\hline Responsibility for harm & $7.5 \pm 5.0$ & $2.6 \pm 2.6$ & 4.8 & $<0.001$ \\
\hline Unacceptable thoughts & $9.5 \pm 5.3$ & $3.1 \pm 3.0$ & 5.8 & $<0.001$ \\
\hline Symmetry, completeness & $5.7 \pm 5.7$ & $2.1 \pm 2.5$ & 3.3 & 0.002 \\
\hline Total & $29.4 \pm 13.0$ & $10.8 \pm 8.0$ & 6.7 & $<0.001$ \\
\hline BDI score & $19.4 \pm 13.4$ & $5.0 \pm 4.5$ & 5.6 & $<0.001$ \\
\hline \multicolumn{5}{|l|}{ Dysfunctional beliefs measures } \\
\hline \multicolumn{5}{|l|}{ OBQ } \\
\hline Responsibility & $65.6 \pm 23.6$ & $55.6 \pm 12.6$ & 2.1 & 0.011 \\
\hline Importance of thoughts & $50.7 \pm 19.3$ & $33.6 \pm 10.8$ & 4.2 & $<0.001$ \\
\hline \multicolumn{5}{|l|}{ GI } \\
\hline Trait & $67.5 \pm 12.4$ & $56.1 \pm 8.7$ & 4.1 & $<0.001$ \\
\hline State & $33.5 \pm 7.8$ & $25.1 \pm 5.9$ & 4.8 & $<0.001$ \\
\hline Moral & $43.6 \pm 7.2$ & $39.3 \pm 5.7$ & 2.5 & 0.014 \\
\hline
\end{tabular}

*fisher's exact test. OCD: obsessive-compulsive disorder, HC: healthy controls, OCI: Obsessive-Compulsive Inventory, DOCS: Dimensional Obsessive Compulsive Scale, BDI: Beck Depression Inventory, OBQ: Obsessive Beliefs Questionnaire, GI: Guilt Inventory

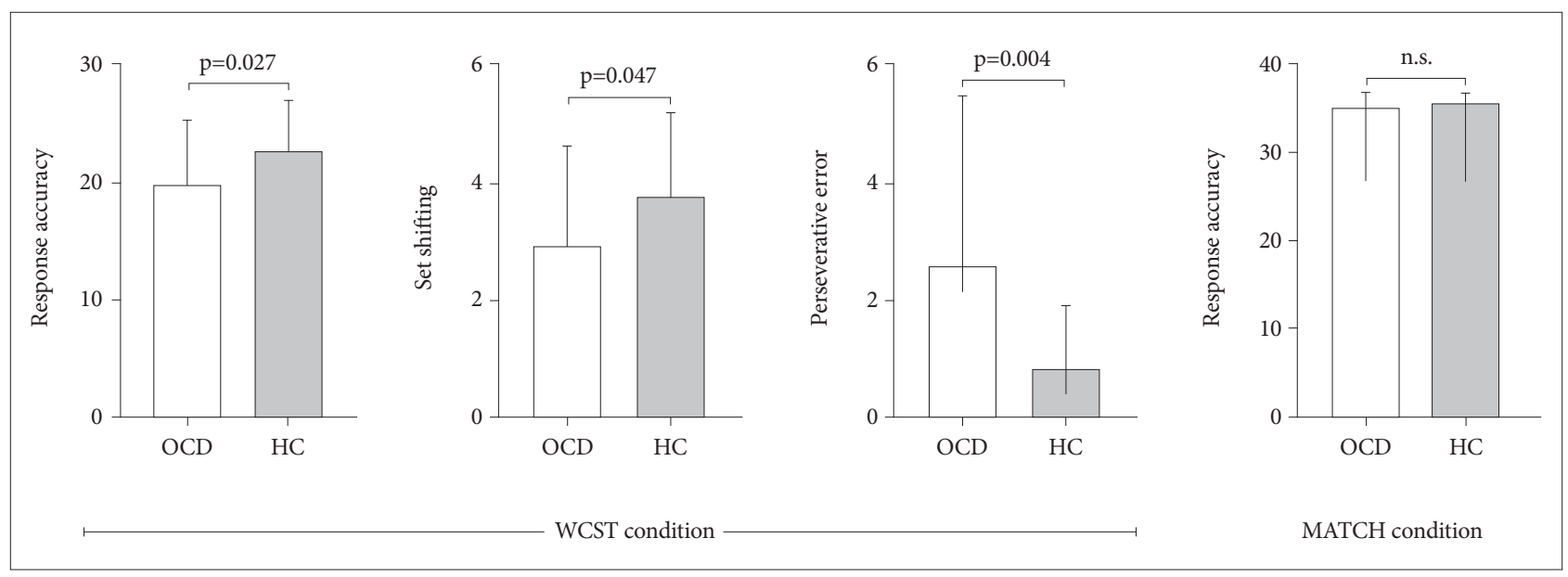

Figure 1. Performance on the Wisconsin Card Sorting Test (WCST) and MATCH condition. OCD: obsessive-compulsive disorder, HC: healthy controls. 


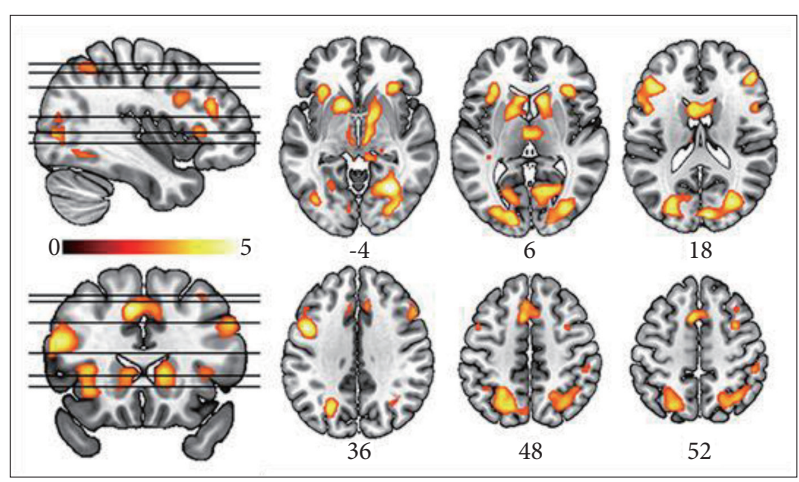

Figure 2. Brain activation for post-hoc analysis of the main effect of condition (Wisconsin Card Sorting Test $>\mathrm{MATCH}$ ). The map was thresholded at false discovery rate corrected $p<0.05$ with a minimum cluster size of 5 .

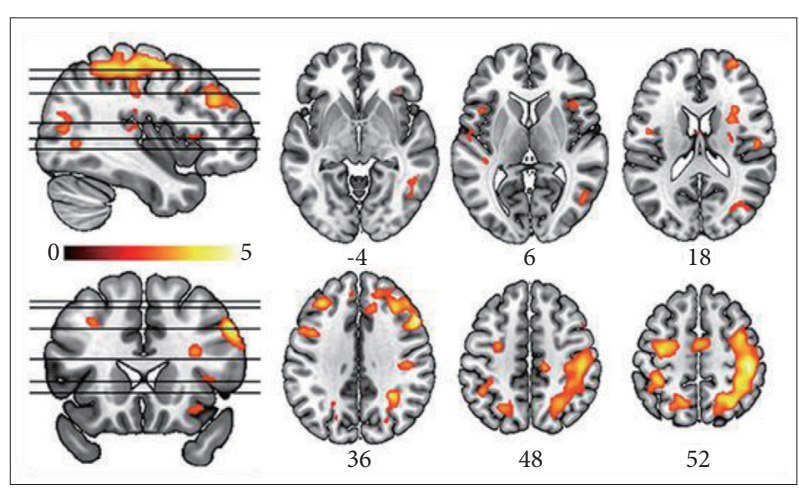

Figure 3. Brain activation for group comparison of the Wisconsin Card Sorting Test condition (obsessive-compulsive disorder>healthy controls). The map was thresholded at false discovery rate corrected $p<0.05$ with a minimum cluster size of 5 .

insula, and bilateral superior and inferior parietal lobules (Figure 4A, Supplementary Table 4 in the online-only Data Supplement).

Among individuals with OCD, three variables of performance on the WCST were not correlated with any brain activity in ROIs.

The subscale of importance of thought on OBQ scale had significant relationships with the right medial frontal gyrus $(\mathrm{r}=0.37, \mathrm{p}=0.04)$ and anterior insula $(\mathrm{r}=0.40, \mathrm{p}=0.03)$, respectively. The score of guilt trait subscale was also significantly correlated with right medial frontal gyrus $(\mathrm{r}=0.39, \mathrm{p}=0.03)$ and inferior orbito-frontal gyrus $(\mathrm{r}=0.46, \mathrm{p}=0.01)$, respectively (Figure $4 \mathrm{~B}$ ). However, no relationship with responsibility subscale of OBQ was found.

In terms of OC dimensions, only the unacceptable thought (pure obsession) dimension was significantly correlated with five out of ten ROIs such as bilateral DLPFC and IPL, and right SPL. However, any other relationships with the other three domains were not found (Supplementary Table 5 in the onlineonly Data Supplement).

\section{WCST performance vs. Dysfunctional beliefs and OC symptom dimensions}

Within the OCD group, three variables of performance on the WCST found no relationships with any dysfunctional belief scales and OC symptom dimensions except the only negative correlation between score of set shifting and unacceptable thought dimension $(\mathrm{r}=-0.43, \mathrm{p}=0.02)$.

\section{DISCUSSION}

To the best of our knowledge, the current study is the first fMRI study to investigate the potential relationship between cognitive deficit (measured by WCST) and dysfunctional belief (measured by scales related to dysfunctional beliefs) mediated by neural activity in OCD. We found that patients with OCD recruited additional frontoparietal regions while performing the WCST task. Several selected ROIs from these additionally activated regions had positive relationships exclusively with dysfunctional beliefs such as importance of thoughts which included TAF and guilt, and with unacceptable thought dimension among four OC symptom dimensions in the OCD group. However, no ROIs showed relationships with variables of performance on the WCST.

This study revealed the engagement of additional frontoparietal network with poorer performance of WCST during MR scan in patients with OCD. First, patients in this study showed lower scores on all three variables of the WCST condition. Poorer performance on WCST, especially perseverative errors, has been confirmed in a series of meta-analysis with approximately 0.5 of effect size. ${ }^{20}$ Second, consistent with previous fMRI studies, our cognitively complex WCST induced activation in a distributed network of brain regions, which includes not only frontoparietal network and posterior multimodal association cortices but also subcortical structures (Figure 2). ${ }^{21,22}$ Our between-group comparison showed additional activation mostly in frontoparietal network in the OCD group. The frontoparietal network, one major subsystem of the control system, is thought to be particularly involved in highly adaptive control processes and has extensive brain-wide connectivity. ${ }^{23}$ Moreover, this system contains flexible hubs, which are updated according to current task demands. ${ }^{24}$ In effect, the evidence for control system alterations has been identified in $\mathrm{OCD}^{25}$ and compensatory frontoparietal brain activity during working memory may constitute a neurocognitive endophenotype for $\mathrm{OCD}^{26}$

Regarding relationships between differentially activated regions and dysfunctional beliefs in patients with OCD, activity in right medial frontal gyrus (namely, the dorsomedial prefrontal cortex; $\mathrm{dmPFC}$ ) and anterior insula were correlated with importance of thoughts. Activation in these two regions 

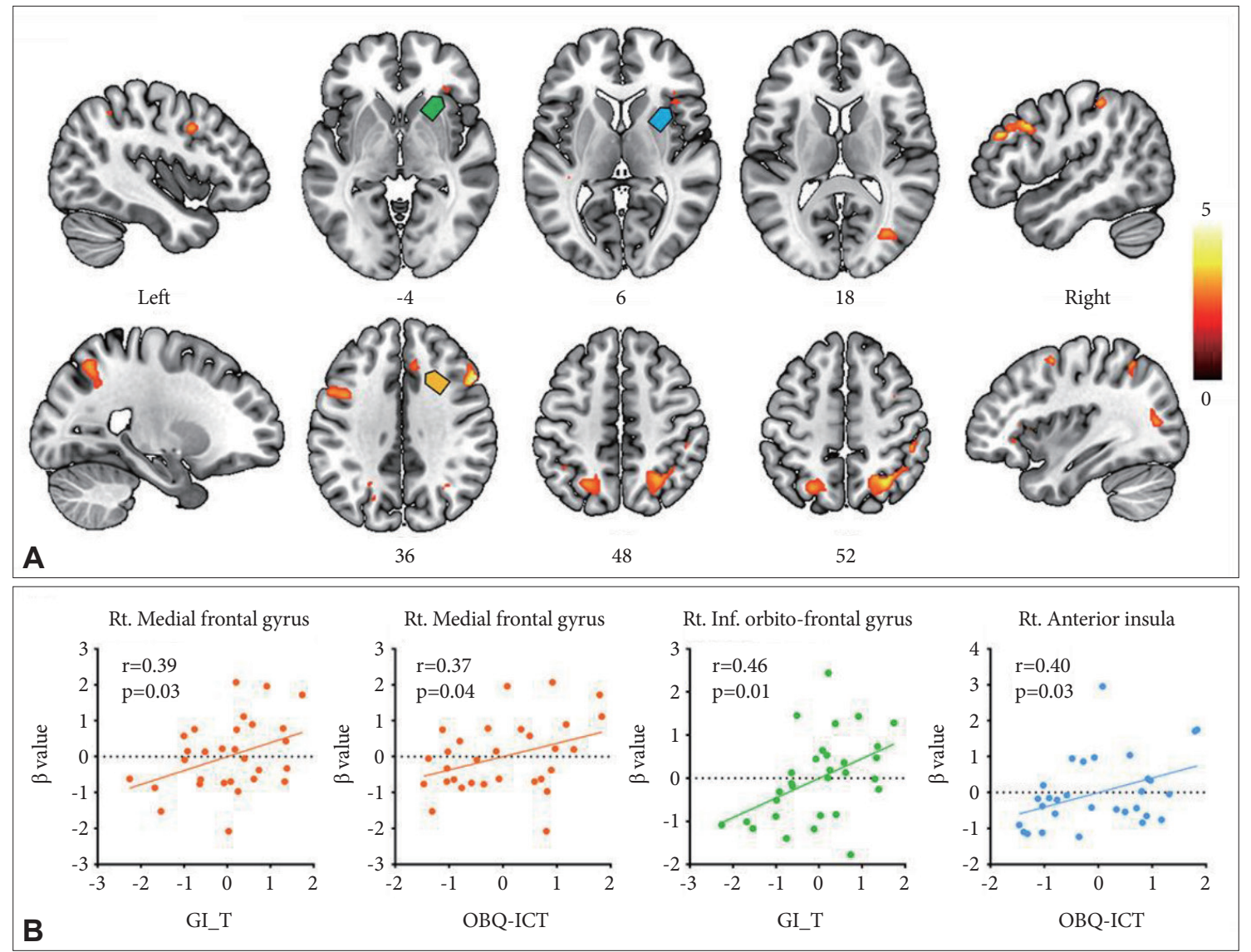

Figure 4. Conjunction map of condition effect (WCST>MATCH) and group effect of WCST condition (obsessive-compulsive disorder>healthy control). Activated brain regions are shown in (A) and correlations between beta activity in some of these regions (green, blue, and orange arrows for right inferior orbito-frontal gyrus, insula, and medial frontal gyrus) and dysfunctional beliefs such as guilt and importance/control of thoughts in (B). WCST: Wisconsin Card Sorting Test, GI-T: Guilt Inventory-Trait, OBQ-ICT: Obsessional Beliefs Questionnaire-Importance/control of thoughts.

were observed in a recent fMRI study using TAF evoking paradigm in healthy participants. ${ }^{27}$ In particular, the right anterior insula may play a role adjusting the intensity of TAF. The anterior insula was, for example, activated more to close person than neutral person in the TAF eliciting statements. Another finding is that feelings of guilt were correlated with activity in the right dmPFC and inferior orbito-frontal gyrus (namely, ventrolateral PFC; vlPFC). Previous studies have revealed guilt-specific activations in the dmPFC and vlPFC..$^{28,29}$ The dmPFC plays a crucial role in self-referential processing ${ }^{30}$ and mentalizing, ${ }^{31}$ whereas the vlPFC is concerned with moral decision-making; patients with OCD showed activation of this region during moral judgment. ${ }^{32}$ Conversely, these activated regions were not correlated with any variables of WCST performance. Simply put, added activation of frontoparietal regions in patients with OCD while performing WCST did not correlate significantly with WCST scores, but rather with dysfunctional beliefs. If we did not see the relationships with dysfunctional beliefs, this study typically would conclude that additional activation of frontoparietal regions might be due to "compensation" of their cognitive dysfunction, like previous studies on cognitive dysfunction in OCD have suggested. ${ }^{26}$ However, these findings were suggestive of another possibility that additional activation may be related not with performance of WCST itself but with dysfunctional beliefs. Patients with OCD may recruit these regions to process accessory beliefs on the performance of the WCST or the whole experiment including the scanning procedures, such that, for example, they would feel guilty if they ruined this experiment or performed tasks poorly. In turn, these additional thoughts or feelings may distract their attention from performance of the task itself and affect their performance.

Another interesting finding is that, among the four OC symptom domains, the unacceptable thoughts domain was the only one that correlated with brain activity in several ROIs exclusively in this study. Moreover, these ROIs were all lateral frontal and parietal cortices, which did not show any correlations with dysfunctional beliefs. In fact, the domain of importance/control of thought, as measured by OBQ-44, showed the greatest correlation with obsessing symptom of $\mathrm{OCI},{ }^{33}$ and 
emerged as the only significant predictor for DOCS unacceptable thoughts scores. ${ }^{34}$ In addition, one recent fMRI study using a TAF induction experiment observed activation in bilateral superior parietal lobules. ${ }^{35}$ However, dysfunctional beliefs can account for all variance in the unacceptable thoughts domain $\left(\mathrm{R}^{2}=0.14\right){ }^{34}$ This finding may explain discrepant relationships of ROIs with dysfunctional beliefs and the unacceptable thoughts domain.

Taken together, among additionally activated frontoparietal areas, medial and inferior frontal portions were related to dysfunctional beliefs while lateral frontal and parietal regions were related to the unacceptable thoughts dimension in patients with OCD. We assumed that the former may produce inner thoughts or feelings with prediction while the latter may offer a system to operate these contents under the context of metacognition (thoughts about thoughts) and self-conscious feelings (feelings about feelings). In particular, these responses may be pronounced in the pure obsessional type of OCD.

There are several limitations to our study. First, dysfunctional beliefs which showed relationships with neural activity were measured using trait-like questionnaires. We did not assess state-dependent thoughts or feelings during MRI scans. Further studies are needed to explore the direct relationship with state-dependent beliefs. Second, our study cannot confirm the direct relationship between two cognitive models since dysfunctional beliefs were only measured by questionnaires, and significance of correlation results was relatively low without multiple correction. However, we believe our results might be a cornerstone to connect two different models for understanding pathophysiology of OCD from an integrative perspective. Third, although one-third of the patients were drugfree during the experiment, the possibility that medication had an effect on our results cannot be completely excluded. ${ }^{36}$ SSRIs can have effects on functional connectivity in OCD patients, ${ }^{37}$ and SSRI treatment can directly associated with function of frontoparietal networks during cognitive task. ${ }^{38}$ Therefore, medication effects can modulate our fMRI results. Fourth, effects of depressive symptoms were not clearly excluded in our results. Although the patterns of group differences were maintained at an uncorrected level, several activations of group differences were attenuated after adjusting for depressive symptoms. Fifth, a male-dominant sample may raise gender issues in this study. However, previous literature has found little evidence for differences in obsessive beliefs between the sexes, suggesting that these beliefs may not be dependent on demographic characteristics. ${ }^{39}$ Lastly, it should be noted that the WCST and MATCH condition as a whole was included in the analysis whether individual trial was correct or not. This is due to several reasons: First, our fMRI task design was block design. Second, WCST inevitably has incorrect trials and re- sponses of incorrect trials can affect followed trials. Third, individual subjects had different correct and incorrect trials, thus amounts of time and data for analysis were inconsistent across subjects.

In conclusion, this fMRI study found that additional activation of frontoparietal regions in patients with OCD while performing the WCST did not correlate with WCST scores, but rather with dysfunctional beliefs and the unacceptable thoughts domain of OCD. These findings suggest that cognitive errors and cognitive deficits may be interrelated in the brain even when performing conventional neurocognitive tasks and coinvolvement of cognitive errors may be pronounced in the unacceptable thoughts domain-dominant OCD patients.

\section{Supplementary Materials}

The online-only Data Supplement is available with this article at https://doi.org/10.30773/pi.2020.0347.

\section{Acknowledgments}

This work was supported by the National Research Foundation of Korea (NRF) grant funded by the Korea government (MSIP) (NRF-2018R1A2B 6007374).

\section{Conflicts of Interest}

The authors have no potential conflicts of interest to disclose.

\section{Author Contributions}

Conceptualization: Sang Won Lee, Seung Jae Lee. Data curation: Seungho Kim, Hyunsil Cha, Eunji Kim, Sang Won Lee. Formal analysis: Seungho Kim, Hyunsil Cha, Eunji Kim. Funding acquisition: Seung Jae Lee. Investigation: Seung Jae Lee. Methodology: Seungho Kim, Sang Won Lee. Project administration: Seung Jae Lee. Resources: Yongmin Chang, Seung Jae Lee. Software: Seungho Kim, Sang Won Lee. Supervision: Yongmin Chang, Seung Jae Lee. Validation: Yongmin Chang, Seung Jae Lee. Visualization: Seungho Kim, Writing—original draft: Seungho Kim, Sang Won Lee, Seung Jae Lee. Writing-review \& editing: Yongmin Chang, Seung Jae Lee, Seungho Kim, Sang Won Lee.

\section{ORCID iDs}

Seungho Kim Sang Won Lee Hyunsil Cha

Eunji Kim Yongmin Chang

Seung Jae Lee

https://orcid.org/0000-0003-4442-7092 https://orcid.org/0000-0002-3537-7110 https://orcid.org/0000-0002-6887-8595 https://orcid.org/0000-0002-2677-4325 https://orcid.org/0000-0002-0585-8714 https://orcid.org/0000-0003-3648-9824

\section{REFERENCES}

1. Taylor S, Abramowitz JS, McKay D, Cuttler C. Cognitive Approaches to Understanding Obsessive Compulsive and Related Disorders. In: Steketee G, Editor. The Oxford Handbook of Obsessive Compulsive and Spectrum Disorders. Oxford: Oxford University Press, 2012, p. 233-250.

2. Hezel DM, McNally RJ. A Theoretical review of cognitive biases and deficits in obsessive-compulsive disorder. Biol Psychol 2016;121:221232.

3. Rachman S, de Silva P. Obsessive-Compulsive Disorder (The Facts Series). 4th Ed. New York: Oxford University Press Inc.; 2009.

4. Gruner P, Pittenger C. Cognitive inflexibility in obsessive-compulsive disorder. Neuroscience 2017;345:243-255. 
5. Shin NY, Lee TY, Kim E, Kwon JS. Cognitive functioning in obsessivecompulsive disorder: a meta-analysis. Psychol Med 2014;44:1121-1130.

6. McNally RJ. On the scientific status of cognitive appraisal models of anxiety disorder. Behav Res Ther 2001;39:513-521.

7. Menzies L, Chamberlain SR, Laird AR, Thelen SM, Sahakian BJ, Bullmore ET. Integrating evidence from neuroimaging and neuropsychological studies of obsessive-compulsive disorder: the orbitofronto-striatal model revisited. Neurosci Biobehav Rev 2008;32:525-549.

8. Abramovitch A, Abramowitz JS, Mittelman A. The neuropsychology of adult obsessive-compulsive disorder: a meta-analysis. Clin Psychol Rev 2013;33:1163-1171.

9. Rachman S. A cognitive theory of obsessions. Behav Res Ther 1997;35: 793-802.

10. Salkovskis PM. Obsessional-compulsive problems: a cognitive-behavioural analysis. Behav Res Ther 1985;23:571-583.

11. Cognitive assessment of obsessive-compulsive disorder. Obsessive Compulsive Cognitions Working Group. Behav Res Ther 1997;35:667-681.

12. Obsessive Compulsive Cognitions Working Group. Development and initial validation of the obsessive beliefs questionnaire and the interpretation of intrusions inventory. Behav Res Ther 2001;39:987-1006.

13. Fisher PL. Obsessive compulsive disorder: a comparison of CBT and the metacognitive approach. Int J Cogn Ther 2009;2:107-122.

14. Goncalves O, Carvalho S, Leite J, Fernandes-Goncalves A, Carracedo A, Sampaio A. Cognitive and emotional impairments in obsessive-compulsive disorder: Evidence from functional brain alterations. Porto Biomed J 2016;1:92-105.

15. Woo CW, Kwon SM, Lim YJ, Shin MS. The Obsessive-Compulsive Inventory-Revised (OCI-R): psychometric properties of the Korean version and the order, gender, and cultural effects. J Behav Ther Exp Psychiatry 2010;41:220-227.

16. Abramowitz JS, Deacon BJ, Olatunji BO, Wheaton MG, Berman NC, Losardo D, et al. Assessment of obsessive-compulsive symptom dimensions: development and evaluation of the Dimensional Obsessive-Compulsive Scale. Psychol Assess 2010;22:180-198.

17. Beck AT, Ward CH, Mendelson M, Mock J, Erbaugh J. An inventory for measuring depression. Arch Gen Psychiatry 1961;4:561-571.

18. Myers SG, Fisher PL, Wells A. Belief domains of the Obsessive Beliefs Questionnaire-44 (OBQ-44) and their specific relationship with obsessive-compulsive symptoms. J Anxiety Disord 2008;22:475-484.

19. Seo J, Chang Y, Jang KE, Park JW, Kim YT, Park SJ, et al. Altered executive function in the welders: a functional magnetic resonance imaging study. Neurotoxicol Teratol 2016;56:26-34.

20. Nyhus E, Barcelo F. The Wisconsin Card Sorting Test and the cognitive assessment of prefrontal executive functions: a critical update. Brain Cogn 2009;71:437-451.

21. Buchsbaum BR, Greer S, Chang WL, Berman KF. Meta-analysis of neuroimaging studies of the Wisconsin card-sorting task and component processes. Hum Brain Mapp 2005;25:35-45.

22. Lie $\mathrm{CH}$, Specht K, Marshall JC, Fink GR. Using fMRI to decompose the neural processes underlying the Wisconsin Card Sorting Test. Neuroimage 2006;30:1038-1049.

23. Cole MW, Repovs G, Anticevic A. The frontoparietal control system: a central role in mental health. Neuroscientist 2014;20:652-664.

24. Marek S, Dosenbach NUF. The frontoparietal network: function, electrophysiology, and importance of individual precision mapping. Dialogues Clin Neurosci 2018;20:133-140.

25. Anticevic A, Hu S, Zhang S, Savic A, Billingslea E, Wasylink S, et al. Global resting-state functional magnetic resonance imaging analysis identifies frontal cortex, striatal, and cerebellar dysconnectivity in obsessive-compulsive disorder. Biol Psychiatry 2014;75:595-605.

26. de Vries FE, de Wit SJ, Cath DC, van der Werf YD, van der Borden V, van Rossum TB, et al. Compensatory frontoparietal activity during working memory: an endophenotype of obsessive-compulsive disorder. Biol Psychiatry 2014;76:878-887.

27. Lee SW, Cha H, Chung Y, Kim E, Song H, Chang Y, et al. The neural correlates of thought-action fusion in healthy adults: A functional magnetic resonance imaging study. Depress Anxiety 2019;36:732-743.

28. Moll J, de Oliveira-Souza R, Garrido GJ, Bramati IE, Caparelli-Daquer EM, Paiva ML, et al. The self as a moral agent: linking the neural bases of social agency and moral sensitivity. Soc Neurosci 2007;2:336-252.

29. Wagner U, N’Diaye K, Ethofer T, Vuilleumier P. Guilt-specific processing in the prefrontal cortex. Cereb Cortex 2011;21:2461-2470.

30. Northoff G, Heinzel A, de Greck M, Bermpohl F, Dobrowolny H, Panksepp J. Self-referential processing in our brain-a meta-analysis of imaging studies on the self. Neuroimage 2006;31:440-457.

31. Isoda $\mathrm{M}$, Noritake $\mathrm{A}$. What makes the dorsomedial frontal cortex active during reading the mental states of others? Front Neurosci 2013;7:232.

32. Harrison BJ, Pujol J, Soriano-Mas C, Hernandez-Ribas R, Lopez-Sola M, Ortiz H, et al. Neural correlates of moral sensitivity in obsessive-compulsive disorder. Arch Gen Psychiatry 2012;69:741-749.

33. Taylor S, Coles ME, Abramowitz JS, Wu KD, Olatunji BO, Timpano KR. How are dysfunctional beliefs related to obsessive-compulsive symptoms? J Cogn Psychother 2010;24:165-176.

34. Wheaton MG, Abramowitz JS, Berman NC, Riemann BC, Hale LR. The relationship between obsessive beliefs and symptom dimensions in obsessive-compulsive disorder. Behav Res Ther 2010;48:949-954.

35. Lee SW, Kim E, Chung Y, Cha H, Song H, Chang Y, et al. Believing is seeing: an fMRI study of thought-action fusion in healthy male adults. Brain Imaging Behav 2021;15:300-310.

36. McCabe C, Mishor Z. Antidepressant medications reduce subcorticalcortical resting-state functional connectivity in healthy volunteers. Neuroimage 2011;57:1317-1123.

37. Shin DJ, Jung WH, He Y, Wang J, Shim G, Byun MS, et al. The effects of pharmacological treatment on functional brain connectome in obsessive-compulsive disorder. Biol Psychiatry 2014;75:606-614.

38. Gyurak A, Petenaude B, Korgaonkar M, Grieve S, Williams L, Etkin A. Frontoparietal activation during response inhibition predicts remission to antidepressants in patients with major depression. Biol Psychiatry 2016;79:274-281.

39. Tripathi A, Avasthi A, Grover S, Sharma E, Lakdawala BM, Thirunavukarasu $\mathrm{M}$, et al. Gender differences in obsessive-compulsive disorder: Findings from a multicentric study from India. Asian J Psychiatr 2018; $37: 3-9$. 
Supplementary Table 1. Pharmacological treatment in patients with OCD $(\mathrm{N}=19)$

\begin{tabular}{|c|c|c|}
\hline Drug & $\mathrm{N}(\%)$ & $\begin{array}{l}\text { Mean daily dosage } \\
\text { (range) }(\mathrm{mg})\end{array}$ \\
\hline Antidepressants & $19(100)$ & \\
\hline ESC & $15(80)$ & $18.3(10-40)$ \\
\hline SER & $1(5)$ & 150 \\
\hline $\mathrm{ESC}+\mathrm{PAR}$ & $1(5)$ & 20,50 \\
\hline ESC+MIR & $1(5)$ & $10,7.5$ \\
\hline BUP+VOR & $1(5)$ & 300,20 \\
\hline Anxiolytics & $14(74)$ & \\
\hline ALP & $4(21)$ & $0.5(0.25-0.75)$ \\
\hline CLO & $3(16)$ & $0.58(0.5-0.75)$ \\
\hline BSP & $2(11)$ & $30(30)$ \\
\hline DZP & $1(5)$ & 4 \\
\hline $\mathrm{ALP}+\mathrm{LRZ}$ & $2(11)$ & $0.5,1(0.5,1)$ \\
\hline $\mathrm{CLO}+\mathrm{DZP}$ & $1(5)$ & 1,2 \\
\hline $\mathrm{ALP}+\mathrm{CLO}$ & $1(5)$ & $0.75,1$ \\
\hline Antipsychotics & $5(26)$ & \\
\hline $\mathrm{ARP}$ & $3(16)$ & $3(2-5)$ \\
\hline RIS & $1(5)$ & 0.5 \\
\hline ARP+RIS & $1(5)$ & 10,1 \\
\hline
\end{tabular}

OCD: obsessive-compulsive disorder, ALP: alprazolam, ARP: aripiprazole, BSP: buspiron, BUP: bupropion, CLO: clonazepam, DZP: diazepam, ESC: escitalopram, LRZ: lorazepam, MIR: mirtazapine, RIS: risperidone, SER: sertraline, VOR: vortioxetine 


\begin{tabular}{|c|c|c|c|c|c|}
\hline \multirow{2}{*}{ Region } & \multirow{2}{*}{ Cluster size } & \multicolumn{3}{|c|}{ Coordinates $(\mathrm{mm})$} & \multirow{2}{*}{ Peak T } \\
\hline & & $\mathrm{x}$ & y & $\mathrm{z}$ & \\
\hline \multicolumn{6}{|l|}{ Superior parietal lobule } \\
\hline $\mathrm{L}$ & 465 & -26 & -58 & 44 & 4.36 \\
\hline $\mathrm{R}$ & 185 & 32 & -64 & 50 & 3.73 \\
\hline \multicolumn{6}{|l|}{ Inferior parietal lobule } \\
\hline $\mathrm{L}$ & 162 & -26 & -58 & 42 & 4.28 \\
\hline $\mathrm{R}$ & 65 & 50 & -34 & 50 & 3.29 \\
\hline \multicolumn{6}{|l|}{ Precuneus } \\
\hline $\mathrm{L}$ & 49 & -12 & -70 & 48 & 3.33 \\
\hline \multicolumn{6}{|l|}{ Supplementary motor area } \\
\hline $\mathrm{L}$ & 299 & -8 & 20 & 44 & 4.80 \\
\hline $\mathrm{R}$ & 53 & 6 & 16 & 50 & 3.54 \\
\hline \multicolumn{6}{|l|}{ Medial frontal gyrus } \\
\hline $\mathrm{L} / \mathrm{R}$ & 187 & -6 & 24 & 44 & 4.89 \\
\hline \multicolumn{6}{|l|}{ Middle frontal gyrus } \\
\hline $\mathrm{L}$ & 87 & -46 & 10 & 36 & 4.44 \\
\hline $\mathrm{R}$ & 198 & 46 & 36 & 20 & 4.70 \\
\hline \multicolumn{6}{|l|}{ Inferior frontal gyrus } \\
\hline $\mathrm{L}$ & 1,017 & -46 & 8 & 24 & 5.66 \\
\hline $\mathrm{R}$ & 508 & 46 & 36 & 24 & 4.37 \\
\hline \multicolumn{6}{|c|}{ Inferior orbito-frontal gyrus } \\
\hline $\mathrm{L}$ & 28 & -34 & 26 & -4 & 4.09 \\
\hline $\mathrm{R}$ & 31 & 32 & 28 & -6 & 3.92 \\
\hline Anterior cingulate cortex & & & & & \\
\hline $\mathrm{L}$ & 75 & -10 & 28 & 26 & 4.14 \\
\hline Precentral gyrus & & & & & \\
\hline $\mathrm{L}$ & 482 & -46 & 6 & 22 & 5.25 \\
\hline $\mathrm{R}$ & 23 & 44 & 8 & 30 & 3.60 \\
\hline Corpus callosum & & & & & \\
\hline $\mathrm{L} / \mathrm{R}$ & 143 & 0 & 8 & 20 & 4.31 \\
\hline Posterior cingulate cortex & & & & & \\
\hline $\mathrm{L}$ & 57 & -20 & -68 & 6 & 3.70 \\
\hline $\mathrm{R}$ & 175 & 20 & -64 & 8 & 4.54 \\
\hline Putamen & & & & & \\
\hline $\mathrm{L}$ & 402 & -16 & 12 & -2 & 4.91 \\
\hline $\mathrm{R}$ & 46 & 18 & 12 & 0 & 3.46 \\
\hline Caudate & & & & & \\
\hline $\mathrm{L}$ & 274 & -12 & 12 & 0 & 4.87 \\
\hline $\mathrm{R}$ & 304 & 10 & 16 & 4 & 4.79 \\
\hline Lingual gyrus & & & & & \\
\hline $\mathrm{L}$ & 167 & -24 & -58 & -2 & 3.23 \\
\hline $\mathrm{R}$ & 411 & 10 & -66 & 4 & 4.39 \\
\hline Insula & & & & & \\
\hline $\mathrm{L}$ & 281 & -32 & 28 & 2 & 4.56 \\
\hline $\mathrm{R}$ & 142 & 32 & 26 & 4 & 4.42 \\
\hline Thalamus & & & & & \\
\hline $\mathrm{L}$ & 100 & -4 & -20 & 2 & 3.72 \\
\hline $\mathrm{R}$ & 166 & 6 & -18 & 0 & 4.26 \\
\hline Medial globus pallidus & & & & & \\
\hline $\mathrm{R}$ & 46 & 12 & 2 & 0 & 5.30 \\
\hline Superior occipital gyrus & & & & & \\
\hline $\mathrm{L}$ & 299 & -16 & -90 & 10 & 5.06 \\
\hline $\mathrm{R}$ & 286 & 28 & -72 & 20 & 4.62 \\
\hline Middle occipital gyrus & & & & & \\
\hline $\mathrm{L}$ & 671 & -18 & -90 & 10 & 5.38 \\
\hline $\mathrm{R}$ & 463 & 32 & -82 & 2 & 4.68 \\
\hline Inferior occipital gyrus & & & & & \\
\hline $\mathrm{L}$ & 130 & -38 & -68 & -10 & 4.73 \\
\hline $\mathrm{R}$ & 53 & 36 & -64 & -10 & 3.95 \\
\hline Hippocampus & & & & & \\
\hline $\mathrm{R}$ & 16 & 18 & -28 & -8 & 3.13 \\
\hline Fusiform & & & & & \\
\hline $\mathrm{L}$ & 334 & -30 & -50 & -18 & 5.09 \\
\hline $\mathrm{R}$ & 342 & 30 & -62 & -6 & 6.25 \\
\hline Cerebellum, anterior lobe & & & & & \\
\hline $\mathrm{L} / \mathrm{R}$ & 452 & -2 & -62 & -32 & 5.77 \\
\hline Cerebellum, posterior lob & & & & & \\
\hline $\mathrm{L} / \mathrm{R}$ & 513 & -2 & -64 & -40 & 5.59 \\
\hline
\end{tabular}

$\mathrm{p}<0.05$, False discovery rate corrected for multiple comparison and minimum cluster size of 5 . 
Supplementary Table 3. Brain activation for group comparison of the Wisconsin Card Sorting Task condition (obsessive-compulsive disorder>healthy controls)

\begin{tabular}{|c|c|c|c|c|c|}
\hline \multirow{2}{*}{ Region } & \multirow{2}{*}{ Cluster size } & \multicolumn{3}{|c|}{ Coordinates (mm) } & \multirow{2}{*}{ Peak T } \\
\hline & & $\mathrm{x}$ & $\mathrm{y}$ & $\mathrm{z}$ & \\
\hline \multicolumn{6}{|l|}{ Superior parietal lobule } \\
\hline $\mathrm{L}$ & 277 & -16 & -58 & 54 & 3.63 \\
\hline $\mathrm{R}$ & 571 & 30 & -58 & 54 & 4.28 \\
\hline \multicolumn{6}{|l|}{ Inferior parietal lobule } \\
\hline $\mathrm{L}$ & 161 & -36 & -40 & 52 & 3.90 \\
\hline $\mathrm{R}$ & 449 & 46 & -34 & 52 & 4.27 \\
\hline \multicolumn{6}{|l|}{ Precuneus } \\
\hline $\mathrm{L}$ & 52 & -12 & -60 & 56 & 3.57 \\
\hline $\mathrm{R}$ & 39 & 14 & -56 & 60 & 3.23 \\
\hline \multicolumn{6}{|l|}{ Supplementary motor area } \\
\hline $\mathrm{L}$ & 60 & 0 & -8 & 54 & 3.60 \\
\hline $\mathrm{R}$ & 96 & 4 & -6 & 56 & 3.67 \\
\hline \multicolumn{6}{|l|}{ Medial frontal gyrus } \\
\hline $\mathrm{L}$ & 59 & -10 & 40 & 32 & 3.29 \\
\hline \multicolumn{6}{|l|}{ Superior frontal gyrus } \\
\hline $\mathrm{L}$ & 153 & -26 & -8 & 62 & 4.69 \\
\hline $\mathrm{R}$ & 343 & 26 & 0 & 62 & 5.09 \\
\hline \multicolumn{6}{|l|}{ Middle frontal gyrus } \\
\hline $\mathrm{L}$ & 217 & -32 & 34 & 38 & 3.98 \\
\hline $\mathrm{R}$ & 884 & 52 & 16 & 40 & 4.64 \\
\hline \multicolumn{6}{|l|}{ Inferior frontal gyrus } \\
\hline $\mathrm{L}$ & 97 & -48 & 8 & 38 & 3.89 \\
\hline $\mathrm{R}$ & 296 & 52 & 18 & 38 & 4.94 \\
\hline \multicolumn{6}{|c|}{ Inferior orbito-frontal gyrus } \\
\hline $\mathrm{R}$ & 17 & 32 & 22 & -12 & 3.22 \\
\hline \multicolumn{6}{|l|}{ Precentral gyrus } \\
\hline $\mathrm{L}$ & 471 & -26 & -10 & 64 & 4.68 \\
\hline $\mathrm{R}$ & 568 & 34 & -10 & 62 & 4.74 \\
\hline \multicolumn{6}{|l|}{ Postcentral gyrus } \\
\hline $\mathrm{L}$ & 125 & -36 & -40 & 54 & 3.92 \\
\hline $\mathrm{R}$ & 874 & 46 & -26 & 54 & 4.82 \\
\hline \multicolumn{6}{|l|}{ Angular gyrus } \\
\hline $\mathrm{R}$ & 142 & 30 & -50 & 38 & 4.11 \\
\hline \multicolumn{6}{|l|}{ Supramarginal gyrus } \\
\hline $\mathrm{L}$ & 24 & -50 & -22 & 24 & 3.82 \\
\hline $\mathrm{R}$ & 46 & 52 & -16 & 24 & 4.07 \\
\hline \multicolumn{6}{|l|}{ Corpus callosum } \\
\hline $\mathrm{L} / \mathrm{R}$ & 59 & -6 & 0 & 26 & 3.58 \\
\hline \multicolumn{6}{|l|}{ Superior temporal gyrus } \\
\hline $\mathrm{L}$ & 48 & -54 & -14 & 0 & 3.28 \\
\hline \multicolumn{6}{|l|}{ Middle temporal gyrus } \\
\hline $\mathrm{R}$ & 291 & 48 & -70 & 12 & 3.79 \\
\hline Insula & & & & & \\
\hline $\mathrm{R}$ & 18 & 40 & 18 & 4 & 3.04 \\
\hline Superior occipital gyrus & & & & & \\
\hline $\mathrm{R}$ & 95 & 26 & -62 & 44 & 3.45 \\
\hline Middle occipital gyrus & & & & & \\
\hline $\mathrm{L}$ & 15 & -24 & -58 & 38 & 3.11 \\
\hline $\mathrm{R}$ & 80 & 44 & -74 & 12 & 3.45 \\
\hline Cerebellum, posterior lob & & & & & \\
\hline $\mathrm{L}$ & 45 & -24 & -72 & -42 & 3.29 \\
\hline
\end{tabular}

$\mathrm{p}<0.05$, False discovery rate corrected for multiple comparison and minimum cluster size of 5 . 
Supplementary Table 4. Conjunction map between condition effect (WCST>MATCH) and group effect of WCST condition (obsessivecompulsive disorder>healthy controls)

\begin{tabular}{|c|c|c|c|c|c|c|}
\hline \multirow{2}{*}{ Region } & \multirow{2}{*}{$\mathrm{BA}$} & \multirow{2}{*}{ Cluster size } & \multicolumn{3}{|c|}{ Coordinates (mm) } & \multirow{2}{*}{ Peak T } \\
\hline & & & $\mathrm{x}$ & $\mathrm{y}$ & $\mathrm{z}$ & \\
\hline \multicolumn{7}{|l|}{ Superior parietal lobule } \\
\hline $\mathrm{L}$ & BA7 & 184 & -24 & -64 & 50 & 3.52 \\
\hline $\mathrm{R}$ & BA7 & 145 & 32 & -58 & 54 & 4.19 \\
\hline \multicolumn{7}{|l|}{ Inferior parietal lobule } \\
\hline $\mathrm{L}$ & BA6 & 5 & -42 & -52 & 48 & 2.83 \\
\hline $\mathrm{R}$ & BA7 & 47 & 40 & -52 & 52 & 3.34 \\
\hline \multicolumn{7}{|l|}{ Medial frontal gyrus } \\
\hline $\mathrm{R}$ & BA8 & 26 & 10 & 28 & 36 & 3.15 \\
\hline \multicolumn{7}{|l|}{ Middle frontal gyrus } \\
\hline $\mathrm{R}$ & BA6 & 39 & 36 & 2 & 56 & 4.24 \\
\hline \multicolumn{7}{|l|}{ Inferior frontal gyrus } \\
\hline $\mathrm{L}$ & BA6 & 91 & -50 & 8 & 38 & 3.81 \\
\hline $\mathrm{R}$ & BA8 & 218 & 50 & 16 & 38 & 4.74 \\
\hline \multicolumn{7}{|c|}{ Inferior orbito-frontal gyrus } \\
\hline $\mathrm{R}$ & $\mathrm{BA} 47$ & 10 & 36 & 30 & -4 & 2.84 \\
\hline \multicolumn{7}{|l|}{ Insula } \\
\hline $\mathrm{R}$ & BA13 & 9 & 38 & 20 & 6 & 2.98 \\
\hline
\end{tabular}

Minimum cluster size of 5. WCST: Wisconsin Card Sorting Task 
Supplementary Table 5. Correlation between WCST-related activated regions and obsessive-compulsive symptom dimensions in the OCD group

\begin{tabular}{|c|c|c|c|c|c|c|c|c|c|c|}
\hline Symptom dimension & Rt. MeFG & Rt. MiFG & Lt. IFG & Rt. IFG & Rt. IOFG & Rt. Insula & Lt. SPL & Rt. SPL & Lt. IPL & Rt. IPL \\
\hline \multicolumn{11}{|l|}{ Germs and contamination } \\
\hline $\mathrm{r}$ & 0.06 & -0.03 & 0.17 & -0.04 & 0.11 & -0.13 & 0.14 & -0.04 & -0.10 & 0.01 \\
\hline $\mathrm{p}$ & 0.74 & 0.88 & 0.39 & 0.83 & 0.57 & 0.48 & 0.48 & 0.82 & 0.58 & 0.97 \\
\hline \multicolumn{11}{|c|}{ Responsibility for harm, injury } \\
\hline $\mathrm{r}$ & 0.19 & 0.28 & 0.01 & 0.22 & 0.26 & 0.31 & 0.24 & 0.28 & 0.12 & 0.31 \\
\hline $\mathrm{p}$ & 0.32 & 0.13 & 0.97 & 0.24 & 0.17 & 0.09 & 0.20 & 0.14 & 0.51 & 0.10 \\
\hline \multicolumn{11}{|l|}{ Unacceptable thoughts } \\
\hline $\mathrm{r}$ & 0.33 & 0.30 & 0.42 & 0.42 & 0.19 & 0.14 & 0.23 & 0.46 & 0.44 & 0.44 \\
\hline $\mathrm{p}$ & 0.07 & 0.11 & 0.02 & 0.02 & 0.32 & 0.45 & 0.23 & 0.01 & 0.02 & 0.01 \\
\hline \multicolumn{11}{|l|}{ Symmetry, completeness } \\
\hline $\mathrm{r}$ & -0.07 & -0.09 & -0.04 & -0.11 & -0.19 & -0.02 & -0.13 & -0.09 & -0.04 & 0.01 \\
\hline $\mathrm{p}$ & 0.71 & 0.62 & 0.83 & 0.56 & 0.32 & 0.92 & 0.49 & 0.63 & 0.82 & 0.95 \\
\hline
\end{tabular}

OCD: obsessive-compulsive disorder, MeFG, MiFG and IFG: medial, middle and inferior frontal gyrus, IOFG: inferior orbito-frontal gyrus, SPL and IPL: superior and inferior parietal lobule 


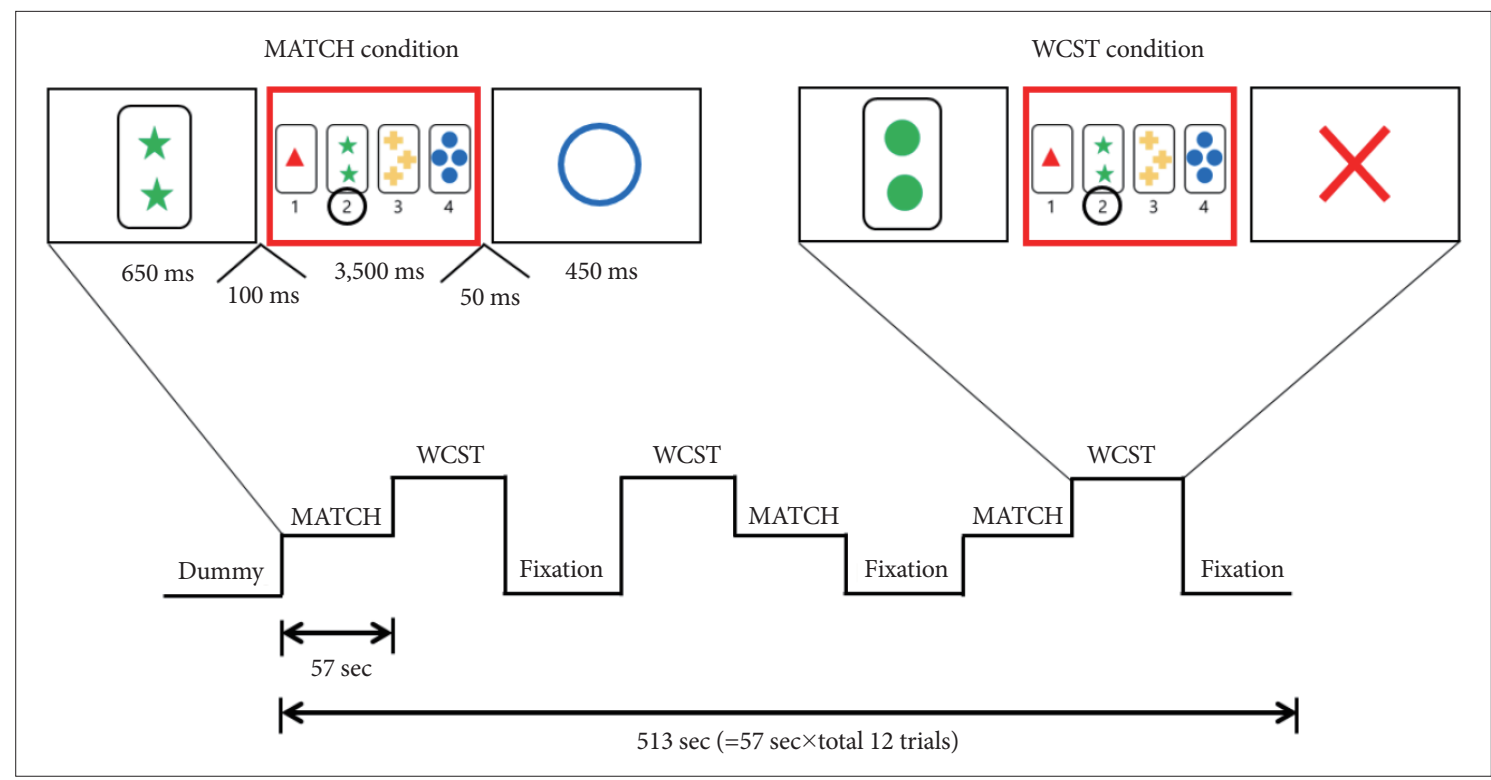

Supplementary Figure 1. The fMRI paradigm of Wisconsin Card Sorting Test (WCST). 


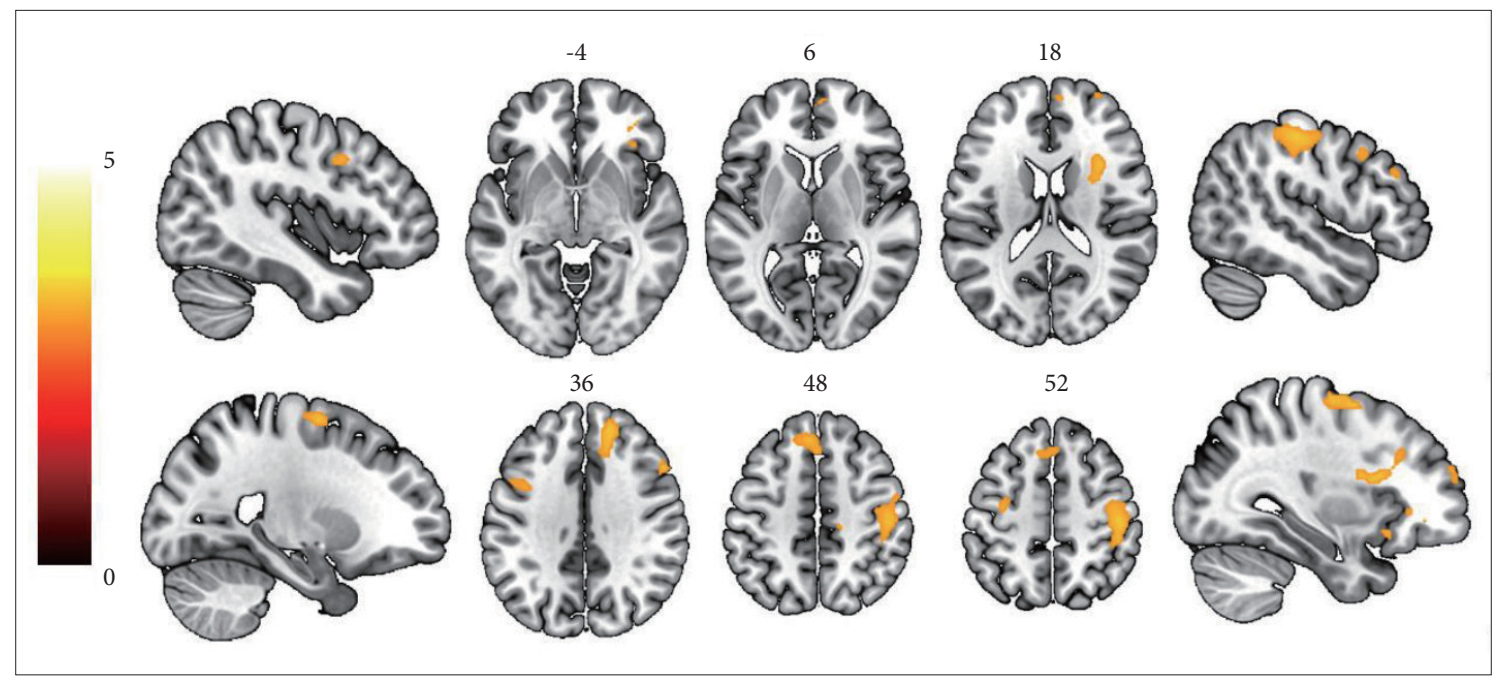

Supplementary Figure 2. Brain activation for group comparison of the Wisconsin Card Sorting Test condition (obsessive-compulsive disorder>healthy controls) after controlling for depressive symptoms. The map was thresholded at uncorrected $p<0.001$ with a minimum cluster size of 5 . 